\title{
NB-IoT and LTE-M towards massive MTC: Complete performance evaluation for $5 \mathrm{G} \mathrm{mMTC}$
}

\author{
Adil Abou El Hassan, Abdelmalek El Mehdi, Mohammed Saber \\ Mohammed First University Oujda, National School of Applied Sciences, SmartICT Lab, Oujda, Morocco
}

\begin{tabular}{|c|c|}
\hline Article Info & ABSTRACT \\
\hline Article history: & \multirow{12}{*}{$\begin{array}{l}\text { Since the emerging } 5 \mathrm{G} \text { wireless network is expected to significantly revolutionize the } \\
\text { field of communication, its standardization and design should regard the internet of } \\
\text { things (IoT) among the main orientations. Also, emerging IoT applications introduce } \\
\text { new requirements other than throughput to support massive machine-type commu- } \\
\text { nication (mMTC) where small data packets are occasionally sent. Therefore, more } \\
\text { importance is attached to coverage, latency, power consumption, and connection den- } \\
\text { sity. For this purpose, the third generation partnership project ( } 3 \mathrm{GPP} \text { ) has introduced } \\
\text { two novel cellular IoT technologies supporting mMTC, known as NB-IoT and LTE- } \\
\text { M. This paper aims to determine the system configuration and deployment required for } \\
\text { NB-IoT and LTE-M technologies to fully meet the } 5 \mathrm{G} \text { mMTC requirements in terms } \\
\text { of coverage, throughput, latency, battery life, and connection density. An overview of } \\
\text { these technologies and their design principles are also described. A complete evalua- } \\
\text { tion of NB-IoT and LTE-M performance against } 5 \mathrm{G} \text { mMTC requirements is presented, } \\
\text { and it is shown that these requirements can be met but only under certain conditions } \\
\text { regarding system configuration and deployment. This is followed by a performance } \\
\text { comparative analysis, which is mainly conducted to determine the limits and suitable } \\
\text { use cases of each technology. }\end{array}$} \\
\hline Received Oct 29, 2020 & \\
\hline Revised May 1, 2021 & \\
\hline Accepted May 15, 2021 & \\
\hline Keywords: & \\
\hline $3 \mathrm{GPP}$ & \\
\hline $5 \mathrm{G}$ & \\
\hline IoT & \\
\hline LTE-M & \\
\hline mMTC & \\
\hline NB-IoT & \\
\hline Performance evaluation & \\
\hline
\end{tabular}

This is an open access article under the CC BY-SA license.

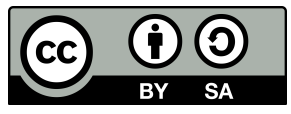

\section{Corresponding Author:}

Adil Abou El Hassan

Mohammed First University Oujda

National School of Applied Sciences

SmartICT Lab, Oujda, Morocco

Email: a.abouelhassan@ump.ac.ma

\section{INTRODUCTION}

Internet of things (IoT) is seen as a driving force behind recent improvements in wireless communication technologies such as third generation partnership project (3GPP), long term evolution advanced (LTE-A) and $5 \mathrm{G}$ new radio (NR) to meet the expected requirements of various massive machine-type communication (mMTC) applications. The mMTC introduces a new communication era where billions of devices, such as remote indoor or outdoor sensors, will need to communicate together while being connected to the cloud-based system.

The purpose of 5G system design is to cover three categories of use cases: enhanced mobile broadband (eMBB), massive machine-type communication (mMTC), as well as ultra reliable low latency communication (uRLLC) which is designed to support critical machine-type communication (cMTC) [1]. The advantage of the $5 \mathrm{G}$ system is the flexibility of its structure, which allows the use of a common integrated system to cover many use cases, by using a new feature which is network slicing based on software-defined networking (SDN) 
and network function virtualization (NFV) technologies [2].

There are currently many low power wide area (LPWA) technologies such as SigFox and LoRa, but their deployment requires new infrastructure implementation with no benefit from existing LTE system. Therefore, 3GPP has introduced in Release 13 (Rel-13) specifications two LPWA technologies for IoT: narrowband IoT (NB-IoT) and LTE machine-type communication (LTE-M(TC)) [3]. The 3GPP Rel-13 core specifications for NB-IoT and LTE-M were finalized in June 2016 [4], [5], while Rel-14 and Rel-15 enhancements were completed in June 2017 and June 2018 respectively [4], [5]. As for Rel-16 enhancements, they were completed in June 2020 whereas Rel-17 enhancements are underway and scheduled for completion in June 2022 [1].

The 3GPP design aims for Rel-13 were low cost and low complexity devices, long battery life, and coverage enhancement to reaching NB-IoT and LTE-M devices in poor coverage conditions. For this purpose, two power saving techniques have been implemented to reduce device power consumption: power saving mode (PSM) and extended discontinuous reception (eDRX) introduced in Rel-12 and Rel-13 respectively [3], [6]. About Rel-15, 3GPP has defined in its work five requirements of 5G mMTC in terms of coverage, throughput, latency, battery life, and connection density [7].

Many papers address 3GPP LPWA technologies including NB-IoT and LTE-M and non-3GPP LPWA technologies such as LoRa and Sigfox. El Soussi et al. [8] implement NB-IoT and LTE-M modules in network simulator NS-3, to evaluate only battery life, latency, and connection density. Whereas Jörke et al. [9] evaluate only throughput, latency, and battery life of NB-IoT and LTE-M. However, Liberg et al. [10] focus on NB-IoT technology only but provide a performance evaluation against 5G mMTC requirements. On the other hand, Krug et al. [11] compare the delay and energy consumption of data transfer covering various IoT communication technologies such as Bluetooth, WiFi, LoRa, Sigfox, and NB-IoT. However, to our knowledge, there is no paper covering the performance evaluation of the NB-IoT and LTE-M technologies against the five requirements of $5 \mathrm{G} \mathrm{mMTC}$ as well as the comparative analysis of these performances.

This paper aims to determine the system configuration and deployment required for NB-IoT and LTE$\mathrm{M}$ technologies to fully meet the $5 \mathrm{G}$ mMTC requirements. Our contribution is to perform a comparative analysis of the performances of NB-IoT and LTE-M technologies, based on the evaluated performances against the $5 \mathrm{G}$ mMTC requirements to determine the limits and suitable use cases of each technology. The remainder of the paper is organized as follows. An overview of the NB-IoT and LTE-M technologies is provided in Section 2. The performance evaluation methodology of NB-IoT and LTE-M technologies is presented in Section 3. This is followed, in Section 4, by a complete performance evaluation of NB-IoT and LTE-M technologies against the 5G mMTC requirements in terms of coverage, throughput, latency, battery life, and connection density. Afterward, a comparative analysis of the evaluated performances of NB-IoT and LTE-M technologies is presented. Also, the enhancements provided by the recent 3GPP releases are discussed. Finally, Section 5 concludes the paper.

\section{OVERVIEW OF CELLULAR IOT TECHNOLOGIES: NB-IOT AND LTE-M}

\subsection{Narrowband IoT: NB-IoT}

The bandwidth occupied by the NB-IoT carrier is $180 \mathrm{kHz}$ corresponding to one physical resource block (PRB) of 12 subcarriers in an LTE system [12]. There are three operation modes to deploy NB-IoT: as a stand-alone carrier, in guard-band of an LTE carrier and in-band within an LTE carrier [13]. To coexist with the LTE system, NB-IoT uses orthogonal frequency division multiple access (OFDMA) in downlink with an identical subcarrier spacing of $15 \mathrm{kHz}$ and frame structure as LTE [14]. Whereas NB-IoT uses in uplink singlecarrier frequency division multiple access (SC-FDMA) and two transmission modes which are the multi-tone and single-tone transmissions to ensure both high capacity and maximum coverage for NB-IoT device with a single antenna [14]. Multi-tone transmission uses the same $15 \mathrm{kHz}$ subcarrier spacing and $0.5 \mathrm{~ms}$ slot duration as LTE, while single-tone transmission supports two numerologies that use $15 \mathrm{kHz}$ and $3.75 \mathrm{kHz}$ subcarrier spacings with $0.5 \mathrm{~ms}$ and $2 \mathrm{~ms}$ slot durations respectively [15]. The restricted quadrature phase-shift keying (QPSK) and binary phase-shift keying (BPSK) modulation schemes are used in both downlink and uplink [16]. Also, NB-IoT defines three coverage enhancement (CE) levels in a cell: CE-0, CE-1, and CE-2 corresponding to the maximum coupling loss (MCL) of $144 \mathrm{~dB}, 154 \mathrm{~dB}$, and $164 \mathrm{~dB}$ respectively [17].

Two device categories Cat-NB1 and Cat-NB2 are defined by NB-IoT which correspond to the device categories introduced in Rel-13 and Rel-14 respectively. The maximum transport block size (TBS) supported in uplink by Cat-NB1 is only 1000 bits compared to 2536 bits for Cat-NB2. Whereas for downlink, the maximum 
TBS supported by Cat-NB1 is only 680 bits compared to 2536 bits for Cat-NB2 [4].

The signals and channels used in downlink (DL) are as follows: narrowband primary synchronization signal (NPSS), narrowband secondary synchronization signal (NSSS), narrowband reference signal (NRS), narrowband physical broadcast channel (NPBCH), narrowband physical downlink shared channel (NPDSCH) and narrowband physical downlink control channel (NPDCCH) [12], [16]. NPDCCH is used to transmit downlink control information (DCI) for uplink, downlink and paging scheduling [12], [16]. In the uplink (UL), only one signal and two channels are used: demodulation reference signal (DMRS), narrowband physical uplink shared channel (NPUSCH) and narrowband physical random access channel (NPRACH). Two formats are used for NPUSCH which are: Format $1(\mathrm{~F} 1)$ and Format 2 (F2). NPUSCH F1 is used by the user equipment (UE) to carry uplink user's data to the evolved node B (eNB), and it supports both single-tone and multi-tone transmissions [17]. Whereas NPUSCH F2 is used to carry uplink control information (UCI), such as hybrid automated repeat request-acknowledgement (HARQ-ACK) and it supports only single-tone transmission [17].

For cell access, the UE must first synchronize with the eNB using NPSS and NSSS signals to achieve time and frequency synchronization with the network and cell identification. Then, it receives narrowband master information block (MIB-NB) and narrowband system information block 1 (SIB1-NB) carried by NPBCH and NPDSCH respectively from eNB to access the system [12], [16].

\subsection{LTE-machine (type communication): LTE-M(TC)}

LTE-M reuses an identical frame structure and also the same numerology as LTE, OFDMA is used in downlink while SC-FDMA is used in uplink with a subcarrier spacing of $15 \mathrm{kHz}$ in both uplink and downlink [18], [19]. The LTE-M transmissions are limited to a narrowband size of 6 PRBs correspondings to $1.4 \mathrm{MHz}$ including guardbands [3]. As the LTE system has a bandwidth from 1.4 to $20 \mathrm{MHz}$, some non-overlapping narrowbands (NBs) can be used if the LTE bandwidth exceeds 1.4 MHz [20]. Up to Rel-14, LTE-M device uses QPSK and 16-QAM modulation schemes with a single antenna for both downlink and uplink. Whereas the support of 64-QAM in downlink has been introduced in Rel-15 [20].

Two device categories are defined by LTE-M: Cat-M1 and Cat-M2 corresponding to device categories introduced in Rel-13 and Rel-14 respectively. Cat-M1 has only a maximum channel bandwidth of $1.4 \mathrm{MHz}$ compared to $5 \mathrm{MHz}$ for Cat-M2 [20]. Besides, Cat-M2 supports a larger TBS of 6968 bits and 4008 bits in uplink and downlink respectively, compared to 2984 bits in both downlink and uplink for Cat-M1 [5].

The following channels and signals are reused by LTE-M in DL: physical downlink shared channel (PDSCH), physical broadcast channel (PBCH), primary synchronization signal (PSS), secondary synchronization signal (SSS), positioning reference signal (PRS), and cell-specific reference signal (CRS). MTC physical downlink control channel (MPDCCH) is the new control channel that has the role of carrying DCI for uplink, downlink and paging scheduling [5], [19]. whereas for UL, the following signals and channels are reused: demodulation reference signal (DMRS), sounding reference signal (SRS), physical uplink shared channel (PUSCH), physical random access channel (PRACH), and physical uplink control channel (PUCCH) which conveys UCI [5], [19].

For cell access, the UE uses the PSS/SSS signals to synchronize with the eNB. Then it uses PBCH which carries the master information block (MIB), and PDSCH which conveys the new system information block 1 for reduced bandwidth UEs (SIB1-BR) from eNB to access the system [19].

\section{METHODOLOGY OF NB-IOT AND LTE-M PERFORMANCE EVALUATION}

The methodology used to perform a complete performance evaluation for both NB-IoT and LTE-M technologies is based on the link level simulations (LLS) as part of 3GPP's works using Ericsson's advanced simulation tool [21-24]. The evaluated performances correspond to the five requirements of 5G $\mathrm{mMTC}$ in terms of coverage defined by the MCL, throughput, latency, battery life, and connection density.

\subsection{Coverage}

The MCL is a common measure to define the level of coverage a system can support. It is depending on the maximum transmitter power $\left(P_{T X}\right)$, the required signal-to-interference-and-noise ratio (SINR), the receiver noise figure $(\mathrm{NF})$, and the signal bandwidth $(\mathrm{BW})[25]$ :

$$
M C L=P_{T X}-\left(S I N R+N F+N_{0}+10 \log _{10}(B W)\right)
$$


where $N_{0}$ is the thermal noise density which is a constant equal to $-174 \mathrm{dBm} / \mathrm{Hz}$ and $P_{T X}$ is referred to as either transmission power per carrier of eNB for downlink MCL calculation or the transmission power of UE for uplink MCL calculation. Whereas the SINR value comes from the LLS and depends on the targeted block error rate (BLER) associated with each channel.

\subsection{Throughput}

The downlink and uplink throughputs of NB-IoT are obtained according to the NPDSCH and NPUSCH F1 transmission time intervals issued from NPDSCH and NPUSCH F1 scheduling cycles respectively and that are provided by the LLS. While the downlink and uplink throughputs of LTE-M are determined based on the PDSCH and PUSCH transmission time intervals issued from PDSCH and PUSCH scheduling cycles respectively and also provided by the LLS. The MAC-layer throughput (THP) is calculated as follows:

$$
T H P=\frac{(1-B L E R)(T B S-O H)}{P D C C H \text { Period }}
$$

It is noteworthy that the TBS of the physical downlink shared channel is used for the downlink THP calculation, whereas the TBS of the physical uplink shared channel is used for the uplink THP calculation. While $\mathrm{OH}$ denotes the overhead size in bits corresponding to the radio protocol stack. Knowing that the periodicity of the user-specific search spaces of physical downlink control channel $\mathrm{T}$ is defined by the product of the relative starting subframe periodicity $(\mathrm{G})$ and the maximum number of repetitions $\left(R_{\max }\right): T=G \times R_{\max }$ [17], [26]. Therefore, the PDCCH period is a multiple of T which corresponds to the periodicity of the scheduled transmissions of physical downlink and uplink shared channels that are used for downlink and uplink THP calculation respectively.

\subsection{Latency}

The latency is defined as the delay between the device synchronization to the cell and the delivery of a data packet to the eNB. It should be evaluated for the following procedures: radio resource control (RRC) Resume procedure and early data transmission (EDT) procedure that has been introduced in Rel-15 and allowing the device to terminate the transmission of small data packets earlier in RRC-idle mode. Figure 1 (a) and Figure 1 (b) depict the data and signaling flows corresponding to the RRC Resume and EDT procedures used by NB-IoT respectively. While the data and signaling flows corresponding to the RRC Resume and EDT procedures used by LTE-M are illustrated in Figure 2 (a) and Figure 2 (b) respectively. The packet definitions and their sizes used for the latency evaluation of NB-IoT and LTE-M at the MCL of $164 \mathrm{~dB}$ are given in Table 1 according to [21].

As shown in Figure 1 (a) and Figure 2 (a), the data packet in RRC Resume procedure is transmitted to the eNB together with the Message 5. Whereas in EDT procedure, the data packet is transmitted to the eNB together with the Message 3 as shown in Figure 1 (b) and Figure 2 (b).

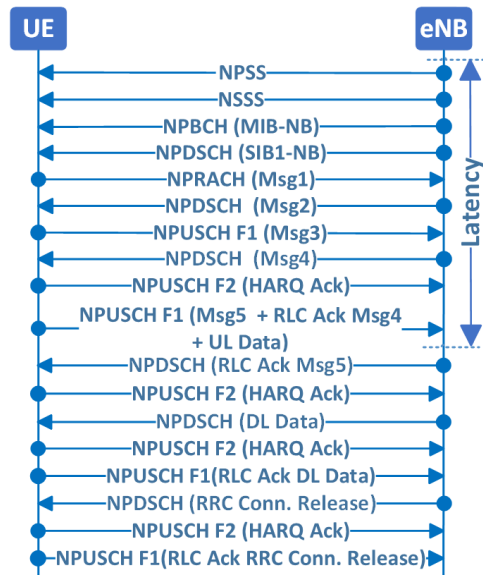

(a)

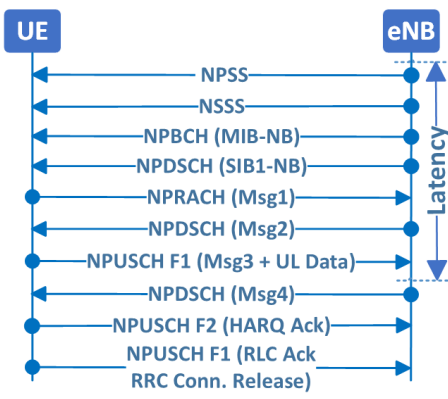

(b)

Figure 1. Data and signaling flows for NB-IoT latency evaluation; (a) RRC resume procedure and (b) EDT procedure 


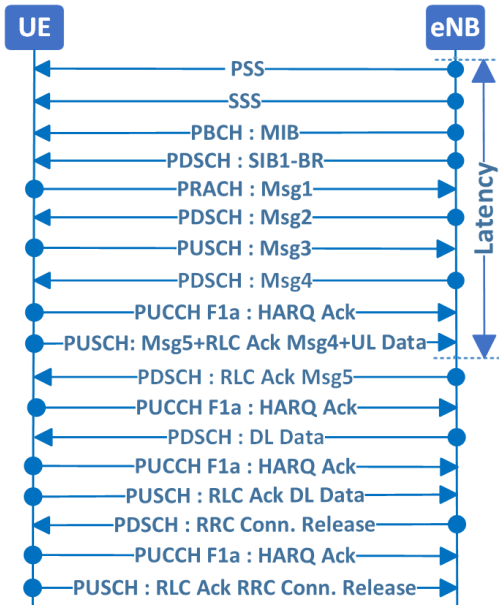

(a)

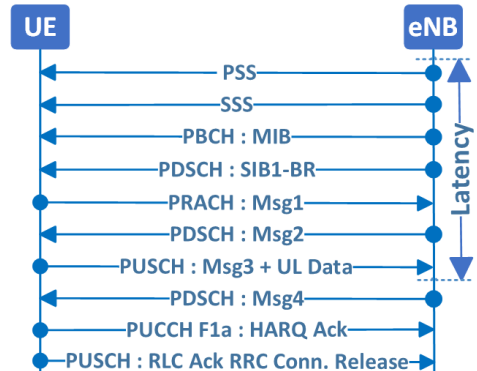

(b)

Figure 2. Data and signaling flows for LTE-M latency evaluation; (a) RRC resume procedure and (b) EDT procedure

Table 1. Packet's definitions and sizes for latency evaluation of NB-IoT and LTE-M

\begin{tabular}{|c|c|c|c|}
\hline \multicolumn{2}{|l|}{ RRC Resume procedure } & \multicolumn{2}{|l|}{ EDT procedure } \\
\hline Random Access Response (Msg2) & 7 bytes & Random Access Response (Msg2) & 7 bytes \\
\hline RRC Conn. Resume (Msg4) & 19 bytes & RRC Conn. Release (Msg4) & $24^{*} \mid 25^{* *}$ bytes \\
\hline + RLC Ack Msg4 + UL report & & & \\
\hline RRC Conn. Release & $17^{*} \mid 18^{* *}$ bytes & & \\
\hline
\end{tabular}

\subsection{Battery life}

The RRC resume procedure is used for battery life evaluation instead of the EDT procedure since EDT procedure does not support uplink TBS larger than 1000 bits which requires long transmission times. The packet flows used to evaluate battery life of NB-IoT and LTE-M are the same as shown in Figure 1 (a) and Figure 2 (a) respectively, where DL data corresponds to the application acknowledgment regarding UL report receipt by eNB. Four levels of device power consumption are defined, including transmission $\left(P_{T x}\right)$, reception $\left(P_{R x}\right)$, Idle-Light sleep $\left(P_{I L S}\right)$ corresponding to the device in RRC-Idle mode or RRC-Connected mode but not actively receiving or transmitting, whereas Idle-Deep sleep $\left(P_{I D S}\right)$ corresponds to power saving mode. The battery life in years is calculated using the following formula according to [27]:

$$
\text { Battery life }[\text { years }]=\frac{\text { Battery energy capacity }}{365 \times \frac{E_{\text {day }}}{3600}}
$$

where $E_{d a y}$ is the device energy consumed per day in Joule and calculated as (4)

$$
E_{d a y}=\left[\left(P_{T x} \times T_{T x}+P_{R x} \times T_{R x}+P_{I L S} \times T_{I L S}\right) \times N_{r e p}\right]+\left(P_{I D S} \times 3600 \times 24\right)
$$

$T_{T x}, T_{R x}$ and $T_{I L S}$ correspond to overall times given in seconds for transmission, reception, and Idle-Light sleep respectively according to packet flows shown in Figure 1 (a) and Figure 2 (a) and obtained from the transmission times of signals and downlink and uplink channels provided by the LLS, while $N_{\text {rep }}$ corresponds to the number of uplink reports per day.

\subsection{Connection density}

The 5G mMTC target on connection density that is also part of the International Mobile Telecommunication targets for 2020 and beyond (IMT-2020), requires the support of one million devices per square kilometer in four different urban macro scenarios [7]. These scenarios are based on two channel models (UMA A) and (UMA B) and two distances of 500 and 1732 meters between adjacent cell sites denoted by ISD (intersite distance) [28]. Based on the simulation assumptions given in Table 2 and the non-full buffer system level 
simulation to evaluate connection density of NB-IoT and LTE-M according to [22], Figure 3 shows the latency required at $99 \%$ reliability to deliver 32 bytes of payload as a function of the connection requests intensity (CRI) to be supported, corresponding to the number of device's connection requests per second, cell and PRB.

Table 2. System level simulation assumptions of urban macro scenarios

\begin{tabular}{ll}
\hline Parameter & Value \\
\hline Frequency band & $700 \mathrm{MHz}$ \\
LTE and LTE-M system bandwidths & $10 \mathrm{MHz}-1.4 \mathrm{MHz}$ \\
Operation mode of NB-IoT & In-band \\
Pathloss model & UMA A, UMA B \\
eNB power and antennas configuration & $46 \mathrm{dBm}-2 \mathrm{Tx} / 2 \mathrm{Rx}$ \\
Device power and antennas configuration & $23 \mathrm{dBm}-1 \mathrm{Tx} / 1 \mathrm{Rx}$ \\
\hline
\end{tabular}

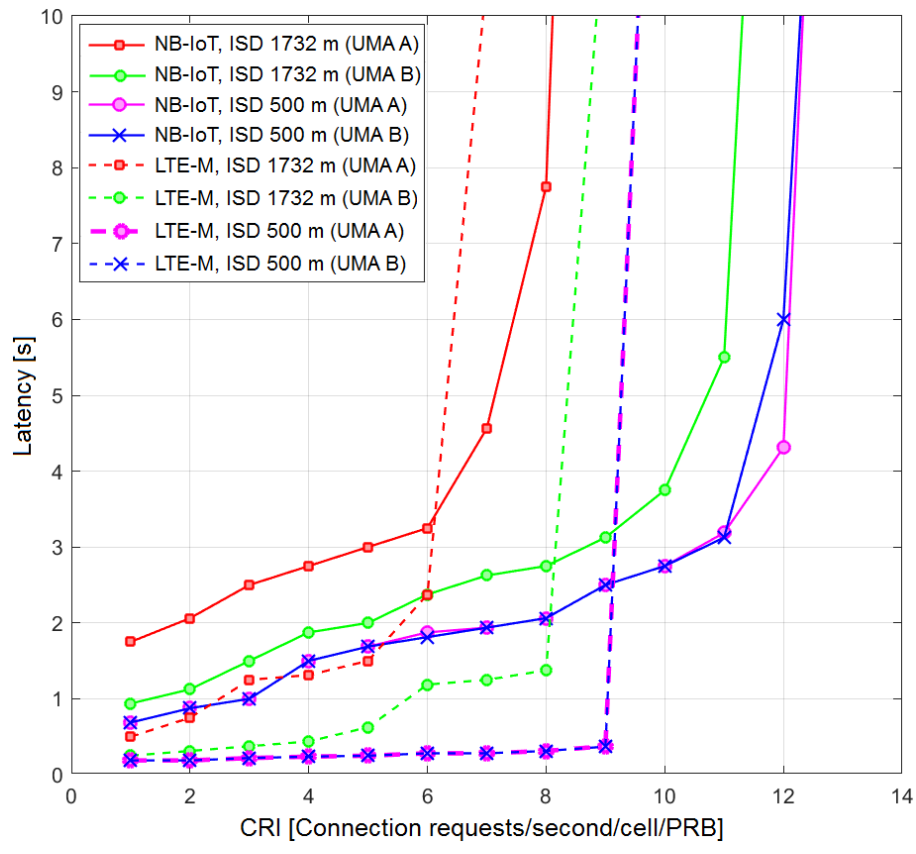

Figure 3. Intensity of connection requests in relation to latency

The latency shown in Figure 3 is evaluated by using the RRC Resume procedure and includes the idle mode time of the device to synchronize to the cell and read the MIB-NB/MIB and SIB1-NB/SIB1-BR.

Knowing that each device must submit a connection request to the system periodically, we can calculate the connection density to be supported (CDS) per cell area using the following formula:

$$
C D S=\frac{C R I \cdot C R P}{A}
$$

where CRP is the periodicity of the device's connection requests given in seconds and the hexagonal cell area A is calculated as follows: $A=I S D^{2} \cdot \sqrt{3} / 6$.

\section{PERFORMANCE EVALUATION RESULTS AND DISCUSSION}

\subsection{Evaluation of NB-IoT and LTE-M performance}

\subsubsection{Coverage}

The simulation assumptions and system model parameters used to evaluate the downlink and uplink MCL are given in Table 3 according to [21]. Based on the simulation assumptions and using (1) to calculate MCL, Table 4 and Table 5 show the NB-IoT and LTE-M channel coverage respectively, to achieve the MCL of $164 \mathrm{~dB}$ which corresponds to the $5 \mathrm{G}$ mMTC coverage requirement to be supported [7]. 
Table 3. Simulation and system model parameters

\begin{tabular}{ll}
\hline Parameter & Value \\
\hline LTE and LTE-M system bandwidths & $10 \mathrm{MHz}-1.4 \mathrm{MHz}$ \\
Channel model / Doppler spread & Tapped Delay Line (TDL-iii NLOS) / 2 Hz \\
NB-IoT mode of operation & Guard-band \\
eNB power and antennas configuration & $46 \mathrm{dBm}-4 \mathrm{Rx} / 2 \mathrm{Tx}$ and 4Rx/4Tx for only (N)PSS/(N)SSS transmissions \\
Device power and antennas configuration & $23 \mathrm{dBm}-1 \mathrm{Rx} / 1 \mathrm{Tx}$ \\
\hline
\end{tabular}

Table 4 and Table 5 also indicate the required acquisition time and block error rate (BLER) associated with each channel to achieve the targeted MCL of $164 \mathrm{~dB}$. From the acquisition times shown in Table 4 and Table 5, we note that to reach the MCL of $164 \mathrm{~dB}$ at the appropriate BLER, it is mandatory to use the time repetition technique for the simulated channels.

Table 4. Downlink and uplink coverage of NB-IoT

\begin{tabular}{lcccccc}
\hline \multirow{2}{*}{$\begin{array}{l}\text { Assumptions } \\
\text { for simulation }\end{array}$} & \multicolumn{2}{c}{ Downlink physical channel } & \multicolumn{3}{c}{ Uplink physical channel } \\
\cline { 2 - 6 } & NPBCH & NPDCCH & NPDSCH & NPRACH & NPUSCH F1 & NPUSCH F2 \\
\hline TBS [Bits] & 24 & 23 & 680 & - & 1000 & 1 \\
Acquisition time [ms] & 1280 & 512 & 1280 & 205 & 2048 & 32 \\
BLER & $10 \%$ & $1 \%$ & $10 \%$ & $1 \%$ & $10 \%$ & $1 \%$ \\
Max transmit power [dBm] & 46 & 46 & 46 & 23 & 23 & 23 \\
Transmit power/carrier [dBm] & 35 & 35 & 35 & 23 & 23 & 23 \\
Noise figure NF [dB] & 7 & 7 & 7 & 5 & 5 & 5 \\
Channel bandwidth [kHz] & 180 & 180 & 180 & 3.75 & 15 & 15 \\
SINR [dB] & -14.5 & -16.7 & -14.7 & -8.5 & -13.8 & -13.8 \\
MCL [dB] & 163.95 & 166.15 & 164.15 & 164.76 & 164 & 164 \\
\hline
\end{tabular}

Table 5. Downlink and uplink coverage of LTE-M

\begin{tabular}{lcccccc}
\hline \multirow{2}{*}{$\begin{array}{l}\text { Assumptions } \\
\text { for simulation }\end{array}$} & \multicolumn{2}{c}{ Downlink physical channel } & \multicolumn{3}{c}{ Uplink physical channel } \\
\cline { 2 - 7 } & PBCH & MPDCCH & PDSCH & PRACH & PUSCH & PUCCH \\
\hline TBS [Bits] & 24 & 18 & 328 & - & 712 & 1 \\
Aquisition time [ms] & 800 & 256 & 768 & 64 & 1536 & 64 \\
BLER & $10 \%$ & $1 \%$ & $2 \%$ & $1 \%$ & $2 \%$ & $1 \%$ \\
Max transmit power [dBm] & 46 & 46 & 46 & 23 & 23 & 23 \\
Transmit power/carrier [dBm] & 39.2 & 36.8 & 36.8 & 23 & 23 & 23 \\
Noise figure NF [dB] & 7 & 7 & 7 & 5 & 5 & 5 \\
Channel bandwidth [kHz] & 945 & 1080 & 1080 & 1048.75 & 30 & 180 \\
SINR [dB] & -17.5 & -20.8 & -20.5 & -32.9 & -16.8 & -26 \\
MCL [dB] & 163.95 & 164.27 & 163.97 & 164.7 & 164 & 165.45 \\
\hline
\end{tabular}

\subsubsection{Throughput}

Figure 4 depicts NPDSCH scheduling cycle of NB-IoT according to [21], where the NPDCCH userspecific search space is configured with a maximum repetition factor $R_{\max }$ of 512 and a relative starting subframe periodicity G of 4 . Whereas the NPUSCH F1 scheduling cycle depicted in Figure 5 corresponds to the scheduling of NPUSCH F1 transmission once every fourth scheduling cycle according to [21].

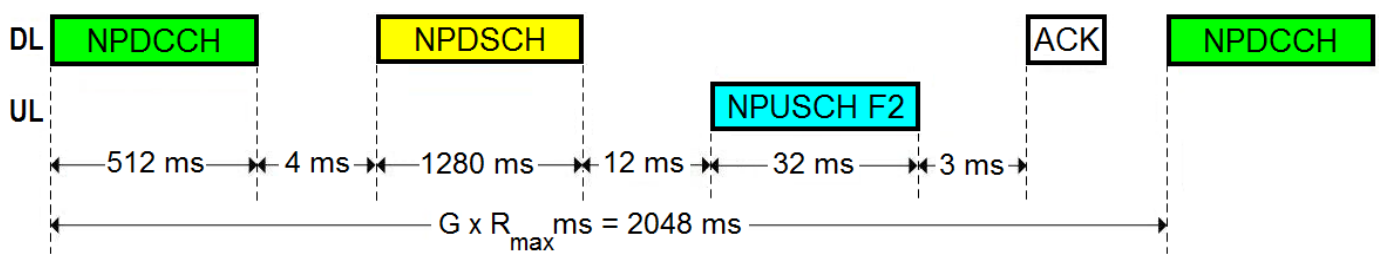

Figure 4. NPDSCH scheduling cycle $\left(R_{\max }=512 ; \mathrm{G}=4\right)$ at the MCL 


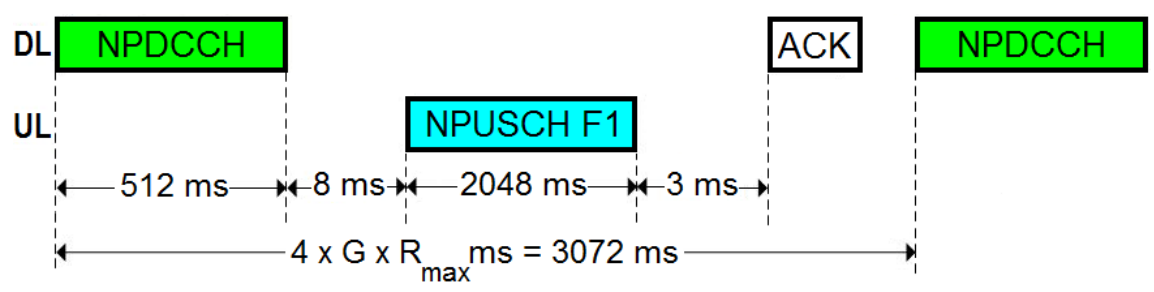

Figure 5. NPUSCH F1 scheduling cycle $\left(R_{\max }=512 ; \mathrm{G}=1.5\right)$ at the MCL

Based on BLER and TBS given in Table 4 and using an overhead $(\mathrm{OH})$ of 5 bytes according to [21], the MAC-layer THP is 281 bps on both downlink and uplink according to the (2). Figure 6 depicts the PDSCH scheduling cycle of LTE-M which corresponds to the scheduling of PDSCH transmission once every third scheduling cycle, where the MPDCCH user-specific search space is configured with $R_{\max }$ of 256 and a relative starting subframe periodicity $\mathrm{G}$ of 1.5 according to [21]. Whereas the PUSCH scheduling cycle depicted in Figure 7 corresponds to the scheduling of PUSCH transmission once every fifth scheduling cycle according to [21].

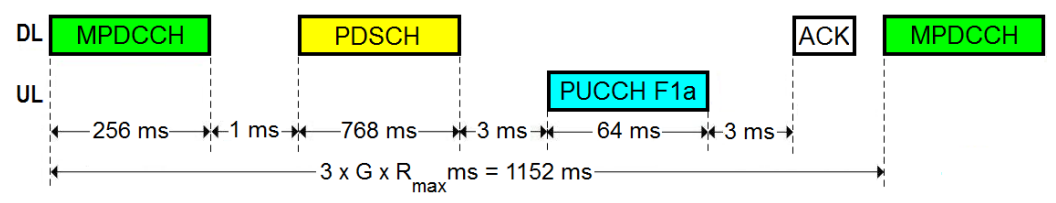

Figure 6. PDSCH scheduling cycle $\left(R_{\max }=256 ; \mathrm{G}=1.5\right)$ at the MCL

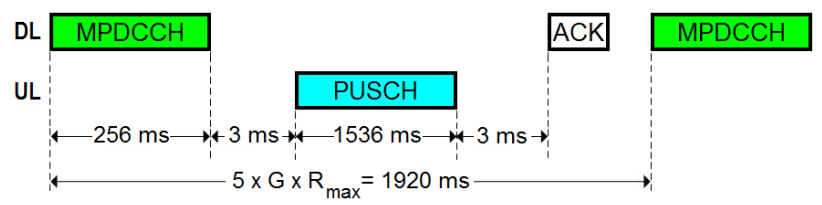

Figure 7. PUSCH scheduling cycle $\left(R_{\max }=256 ; \mathrm{G}=1.5\right)$ at the MCL

From BLER and TBS indicated in Table 5 and the use of an overhead $(\mathrm{OH})$ of 5 bytes, the MAC-layer throughputs obtained in downlink and uplink are $245 \mathrm{bps}$ and $343 \mathrm{bps}$ respectively according to the (2). As part of 3GPP Rel-15, 5G mMTC requires that downlink and uplink troughputs supported at the MCL of $164 \mathrm{~dB}$ must be at least 160 bps [7]. As can be seen, the MAC-layer throughputs of both NB-IoT and LTE-M technologies meet the 5G mMTC requirement, which corresponds to the suitable throughput for IoT applications using sporadic transmissions of small data packets. It should be noted that the BLER targets associated with each NB-IoT and LTE-M channel require the acquisition times shown in Table 4 and Table 5 respectively. Therefore, the throughput levels can be further improved by using the new device categories Cat-NB2 and Cat-M2 which support a larger TBS in both downlink and uplink with enhanced HARQ processes.

\subsubsection{Latency}

The latency evaluation is based on the same system model with the parameters given in Table 3 and using the simulation assumptions relating to the RRC Resume and EDT procedures indicated in Table 1. Using the RRC Resume procedure, the evaluated latency of NB-IoT is 9 seconds, while the EDT procedure allows obtaining a latency of only 5.8 seconds according to [21]. Regarding the latency evaluation of LTE$\mathrm{M}$, the latencies obtained by using the RRC Resume and EDT procedures are 7.7 and 5 seconds respectively. Therefore, the 5G mMTC target of 10 seconds latency at the MCL of $164 \mathrm{~dB}$ defined in 3GPP Rel-15 [7] is met by NB-IoT and LTE-M technologies for both RRC Resume and EDT procedures. However, the best latencies of 5.8 and 5 seconds obtained by NB-IoT and LTE-M respectively using the EDT procedure are mainly due to the multiplexing of the user data with the Message 3 on the dedicated traffic channel, as shown in Figure 1 (b) and Figure 2 (b) respectively. 


\subsubsection{Battery life}

The simulation and system model parameters used to evaluate the battery life of NB-IoT and LTE-M are given in Table 6 according to [23],[24]. While the assumed traffic model according to Rel-14 scenario and device power consumption levels used are given in Table 7 according to [23], [24]. Also, an Active Timer of 20 seconds is included after connection release where the device is in Idle-Light sleep before switching to IdleDeep sleep, to monitor the downlink control channels of NB-IoT and LTE-M i.e. NPDCCH and MPDCCH respectively.

Based on the transmission times of the signals and downlink and uplink channels given in [23] and using the (3) and (4) with the simulation assumptions that are given in Table 7 and a $5 \mathrm{Wh}$ battery, the evaluated battery lives of NB-IoT to achieve the MCL of $164 \mathrm{~dB}$ in in-band, guard-band and stand-alone operation modes are 11.4, 11.6 and 11.8 years respectively. Whereas the evaluated battery life of LTE-M to achieve the MCL of $164 \mathrm{~dB}$ is 8.8 years according to the assumed transmission times given in [24].

To significantly increase the battery life of LTE-M, the uplink throughput should be improved by the increase of the number of base station receiving antennas, thereby reducing UE transmission time. Therefore based on the simulation assumptions given in Table 3 where the number of base station receiving antennas is 4 instead of only 2 according to [21] and the simulation assumptions given in Table 7, the evaluated battery lives of LTE-M and NB-IoT are 11.9 and 11.8 years respectively.

Knowing that the $5 \mathrm{G}$ mMTC requires battery life beyond 10 years at the MCL of $164 \mathrm{~dB}$, supposing an energy storage capacity of 5Wh [7]. Therefore, NB-IoT achieves the targeted battery life in all operation modes regardless of the antennas configuration of the base station. However, LTE-M fulfills the 5G mMTC targeted battery life except if the number of base station receiving antennas is 4 .

Table 6. Simulation and system model parameters for battery life evaluation

\begin{tabular}{ll}
\hline Parameter & Value \\
\hline LTE system bandwidth & $10 \mathrm{MHz}$ \\
Channel model and Doppler spread & Rayleigh fading ETU $-1 \mathrm{~Hz}$ \\
& NB-IoT: 46 dBm (Guard-band, In-band) - 2Tx/2Rx \\
eNB power and antennas configuration & $43 \mathrm{dBm}$ (Stand-alone) - 1Tx/2Rx \\
& LTE-M: 46 dBm - 2Tx/2Rx \\
Device power and antennas configuration & 23 dBm - 1Tx/1Rx \\
\hline
\end{tabular}

Table 7. Traffic model and device power consumption levels

\begin{tabular}{ll}
\hline \multicolumn{2}{c}{ Message format } \\
\hline UL report & 200 bytes \\
DL Application Acknowledgment & 20 bytes \\
UL report periodicity & Once every 24 hours \\
\multicolumn{1}{c}{ Device power consumption } \\
Transmission and reception power consumption & $P_{T x}: 500 \mathrm{~mW}-P_{R x}: 80 \mathrm{~mW}$ \\
Idle mode power consumption & $P_{I L S}: 3 \mathrm{~mW}-P_{I D S}: 0.015 \mathrm{~mW}$ \\
\hline
\end{tabular}

\subsubsection{Connection density}

The supported connection density (CDS) that is evaluated corresponds to the overall number of devices that successfully transmit a payload of 32 bytes accumulated over two hours with the required latency. To evaluate CDS of NB-IoT per PRB and square kilometer depicted in Figure 8 (a), the CDS is calculated from (5) using the CRI values of Figure 3 and periodicity of connection requests of two hours.

Regarding LTE-M, to evaluate CDS per narrowband and square kilometer shown in Figure 8 (b), the CDS is determined from (5) using the CRI values of Figure 3, a reporting period of two hours and scaling of a factor 6 corresponding to the LTE-M narrowband (NB) of 6 PRBs.

In the two scenarios corresponding to the 500 meters ISD shown in Figure 8 (a), more than 1.2 million devices per PRB and square kilometer can be supported by an NB-IoT carrier with a maximum 10 seconds latency. However, only 94000 and 68000 devices per PRB and square kilometer can be supported using the (UMA B) and (UMA A) channel models respectively with an ISD of 1732 meters within the 10-second latency limit. Since, in the scenario of a 1732 meters ISD, the density of base stations is 12 times lower than in a 500 meters ISD. Therefore, this difference in base station density results in differences of up to 18 times between the connection densities relating to the 500 and 1732 meters ISD scenarios. 
As shown in Figure 8 (b), in 500 meters ISD scenario a single narrowband can support up to 5.68 million devices within the 10-second latency limit, by the addition of 2 further PRBs to transmit PUCCH. For the 1732 meters ISD and (UMA B) scenario, the cell size is 12 times larger that explains an LTE-M carrier can only support 445000 devices within the limit of latency of 10 seconds. Also, to further improve the LTE-M connection density, the sub-PRB resource allocation in uplink that has been introduced in 3GPP Rel-15 can be used for low base station density scenarios.

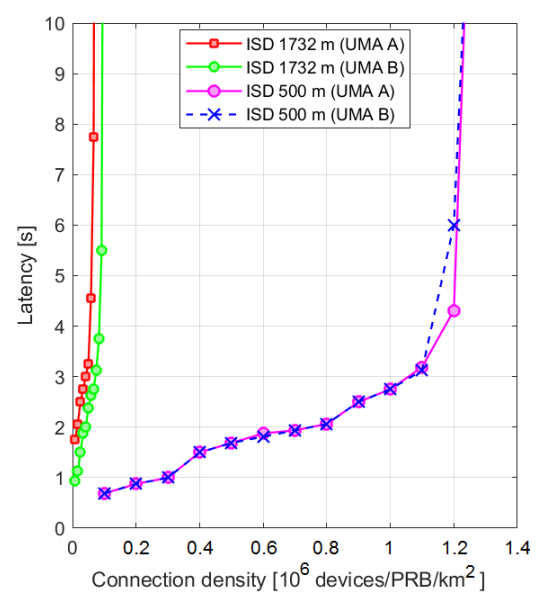

(a)

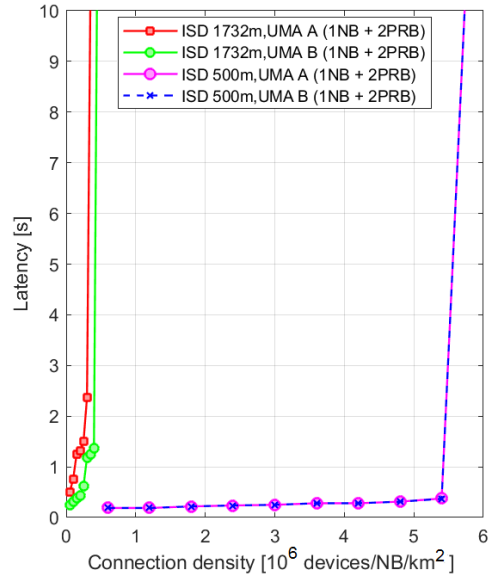

(b)

Figure 8. Connection density in relation to latency of NB-IoT and LTE-M; (a) NB-IoT and (b) LTE-M

\subsection{Comparative analysis of NB-IoT and LTE-M performance}

Figure 9 depicts the diagram comparing the performance of NB-IoT and LTE-M technologies in terms of coverage, throughput, latency, and battery life that have been evaluated in Subsection (4.1.), on using the same simulation assumptions given in Table 3. Whereas the connection densities of NB-IoT and LTE-M that are compared are the ones evaluated using the simulation assumptions given in Table 2. The latencies shown in Figure 9 are that obtained with the EDT procedure, while the connection densities correspond to the best value obtained of the supported intensity of connection requests (CRI) from Figure 3 within the 10-second latency limit, and corresponding to the same urban macro scenario using 500 meters ISD and (UMA B) channel model. The 5G mMTC requirement regarding CRI shown in Figure 9 corresponds to the targeted CRI that is obtained from (5) to achieve one million devices per PRB and square kilometer for 500 meters ISD scenario.

From Table 4 and Table 5, it can be seen that for both technologies, NPUSCH F1 and PUSCH can be considered as the limiting channels, i.e. the channels that need the maximum transmission times to reach the MCL of $164 \mathrm{~dB}$. Indeed, NPDCCH must be configured with 512 repetitions to achieve the targeted BLER of $1 \%$, while the maximum configurable repetition number is 2048 in an extreme coverage corresponding to the CE-2 level [26]. Whereas, MPDCCH needs to be configured with the maximum configurable repetition number, i.e. 256 repetitions to reach the targeted BLER of $1 \%$ and the MCL of $164 \mathrm{~dB}$. Therefore, to support operations in extreme coverage, NB-IoT technology can be considered more efficient than LTE-M technology.

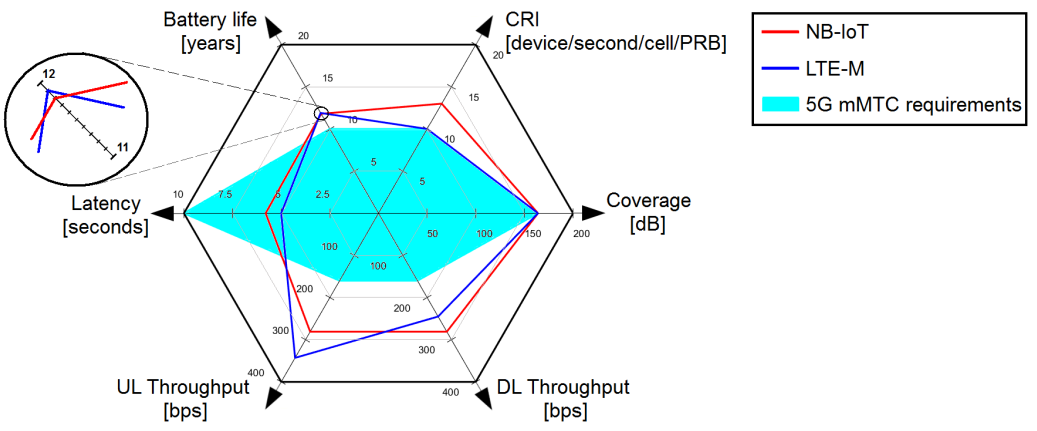

Figure 9. Performance comparison diagram of NB-IoT and LTE-M technologies 
As shown in Figure 9, LTE-M can offer significantly higher uplink throughput due to the larger device bandwidths and reduced processing times. Also, Figure 9 shows that LTE-M performs slightly better than NB-IoT in terms of latency using the EDT procedure. The justification is that MPDCCH achieves the MCL of $164 \mathrm{~dB}$ for a transmission time of $256 \mathrm{~ms}$ according to Table 5, compared to NPDCCH transmission time of $512 \mathrm{~ms}$ according to Table 4. Therefore, LTE-M technology is capable of serving IoT applications requiring relatively short response times such as End-Device positioning and voice over LTE (VoLTE).

LTE-M is seen from Figure 9 to perform slightly more efficiently than NB-IoT in terms of battery life to achieve the MCL of $164 \mathrm{~dB}$, except if the number of base station receiving antennas is 4 instead of only 2 according to [21]. However, to further increase the battery life, MTC Wake-Up Signal (MWUS) and Narrowband WUS (NWUS) introduced in 3GPP Rel-15 can be implemented in LTE-M and NB-IoT devices respectively. These signals allow the UE to remain in idle mode until informed to decode PDCCH/NPDCCH channels for a paging occasion, thereby achieving energy saving.

Figure 9 also indicates that NB-IoT offers a 30 percent higher connection density than LTE-M, which is due to the efficient use of sub-carrier NPUSCH transmissions by using a large number of repetitions under poor coverage conditions. Therefore, NB-IoT is likely to meet IoT applications requiring a massive number of connected devices, such as smart metering systems for electricity, gas, and water consumption measurement.

\section{CONCLUSION}

To conclude, this paper shows that the five targets of 5G mMTC are achieved by both NB-IoT and LTE-M technologies. However, the results of performance evaluation show that the performances are achieved except under certain conditions regarding system configuration and deployment, such as the number of repetitions for channels transmission, the number of base station antennas, and the density of base stations, which is highly dependent on inter-site distance. Regarding the coverage and connection density, NB-IoT offers better performances than LTE-M, while LTE-M performs more efficiently than NB-IoT in terms of throughput, latency, and battery life. Therefore, NB-IoT is the best performing technology for IoT applications supporting operations in extreme coverage and a massive number of devices. Whereas LTE-M is the most efficient technology that meets the requirements of IoT applications needing relatively shorter response times. Further study may be conducted by using simulations based on the recent enhancements introduced in 3GPP Rel-15, such as the use of NWUS/MWUS signals and sub-PRB resource allocation for LTE-M uplink, to validate the performance improvement of NB-IoT and LTE-M technologies against 5G mMTC requirements.

\section{REFERENCES}

[1] A. Ghosh, A. Maeder, M. Baker and D. Chandramouli, "5G Evolution: A View on 5G Cellular Technology Beyond 3GPP Release 15,” IEEE Access, vol. 7, pp. 127639-127651, 2019, doi: 10.1109/ACCESS.2019.2939938.

[2] A. A. Barakabitze, A. Ahmad, R. Mijumbi, and A. Hines, "5G network slicing using SDN and NFV: A survey of taxonomy, architectures and future challenges," Computer Networks, vol. 167, 106984, 2020, doi: 10.1016/j.comnet.2019.106984.

[3] N. L. Ismail, M. Kassim, Ma. Ismail, and R. Mohamad "A review of low power wide area technology in licensed and unlicensed spectrum for IoT use cases," Bulletin of Electrical Engineering and Informatics (BEEI), vol. 7, no. 2, pp. 183-190, Jun 2018, doi: 10.11591/eei.v7i2.1174.

[4] R. Ratasuk, N. Mangalvedhe, Z. Xiong, M. Robert and D. Bhatoolaul, "Enhancements of narrowband IoT in 3GPP Rel-14 and Rel-15," in 2017 IEEE Conference on Standards for Communications and Networking (CSCN), 2017, pp. 60-65, doi: 10.1109/CSCN.2017.8088599.

[5] R. Ratasuk, N. Mangalvedhe, D. Bhatoolaul and A. Ghosh, "LTE-M Evolution Towards 5G Massive MTC," in 2017 IEEE Globecom Workshops (GC Wkshps), 2017, pp. 1-6, doi: 10.1109/GLOCOMW.2017.8269112..

[6] A. K. Sultania, P. Zand, C. Blondia and J. Famaey, "Energy Modeling and Evaluation of NB-IoT with PSM and eDRX," in 2018 IEEE Globecom Workshops (GC Wkshps), 2018, pp. 1-7, doi: 10.1109/GLOCOMW.2018.8644074.

[7] 3GPP TR 38.913, "5G: Study on scenarios and requirements for next generation access technologies Release 15," version 15.0.0, Sep 2018. [Online]. Available: https://www.etsi.org/deliver/etsi_tr/138900_138999/138913/15.00.00_60/tr_138913v150000p.pdf

[8] M. El Soussi, P. Zand, F. Pasveer and G. Dolmans, "Evaluating the Performance of eMTC and NB-IoT for Smart City Applications," in 2018 IEEE International Conference on Communications (ICC), 2018.

[9] P. Jörke, R. Falkenberg and C. Wietfeld, "Power Consumption Analysis of NB-IoT and eMTC in Challenging Smart City Environments," in 2018 IEEE Globecom Workshops (GC Wkshps), 2018, pp. 1-6. 
[10] O. Liberg et al., "Narrowband Internet of Things 5G Performance," in 2019 IEEE 90th Vehicular Technology Conference (VTC2019-Fall), 2019, pp. 1-5, doi: 10.1109/VTCFall.2019.8891588.

[11] S. Krug and M. O'Nils, "Modeling and Comparison of Delay and Energy Cost of IoT Data Transfers," IEEE Access, vol. 7, pp. 58654-58675, 2019, doi: 10.1109/ACCESS.2019.2913703.

[12] L. Feltrin, et al., "Narrowband IoT: A Survey on Downlink and Uplink Perspectives," IEEE Wireless Communications, vol. 26, no. 1, pp. 78-86, February 2019, doi: 10.1109/MWC.2019.1800020.

[13] A. Ali, G. A.Shah, M. O. Farooq, and U. Ghani, "Technologies and challenges in developing machine-tomachine applications: A survey," Journal of Network and Computer Applications, vol. 83, pp. 124-139, 2017, doi: 10.1016/j.jnca.2017.02.002.

[14] C. B. Mwakwata, H. Malik, M. M. Alam, Y. L. Moullec, S. Parand, and S. Mumtaz, "Narrowband Internet of Things (NB-IoT): From physical (PHY) and media access control (MAC) layers perspectives," Sensors, vol. 19, no. 11, pp. 2613, 2019, doi: 10.3390/s19112613.

[15] Y.-P. E. Wang, et al., "A Primer on 3GPP Narrowband Internet of Things," IEEE Communications Magazine, vol. 55, no. 3, pp. 117-123, March 2017, doi: 10.1109/MCOM.2017.1600510CM.

[16] A. Mahmood and S. Zafar, "Performance Analysis of Narrowband Internet of Things (NB-IoT) Deployment Modes," in 2019 22nd International Multitopic Conference (INMIC), 2019, pp. 1-8, doi: 10.1109/INMIC48123.2019.9022748.

[17] E. Rastogi, N, Saxena, A. Roy, and D. R. Shin, "Narrowband Internet of Things: A Comprehensive Study," Computer Networks, vol. 173, no. 107209, 2020, doi: 10.1016/j.comnet.2020.107209.

[18] A. Z. Yonis and M. F. L. Abdullah, "Uplink and Downlink of LTE-Release 10 in Cellular Communications," International Journal of Informatics and Communication Technology (IJ-ICT), vol. 1, no. 1, pp. 43-53, July 2012, doi: 10.11591/ij-ict.v1i1.557.

[19] A. Rico-Alvarino, et al., "An overview of 3GPP enhancements on machine to machine communications," IEEE Communications Magazine, vol. 54, no. 6, pp. 14-21, June 2016, doi: 10.1109/MCOM.2016.7497761.

[20] S.R. Borkar, "Long-term evolution for machines (LTE-M)," in LPWAN Technologies for IoT and M2M Applications, Academic Press, pp. 145-166, 2020, doi: 10.1016/B978-0-12-818880-4.00007-7.

[21] Ericsson, R1-1907398, "IMT-2020 self evaluation: mMTC coverage, data rate, latency \& battery life," 3GPP TSG-RAN WG1 Meeting \#97, Reno, USA, May 2019. [Online]. Available: https://www.3gpp.org/ftp/TSG_RAN/WG1_RL1/TSGR1_97/Docs/R1-1907398.zip

[22] Ericsson, R1-1907399, "IMT-2020 self evaluation: mMTC non-full buffer connection density for LTEMTC and NB-IoT," 3GPP TSG-RAN WG1 Meeting \#97, Reno, USA, May 2019. [Online]. Available: https://www.3gpp.org/ftp/TSG_RAN/WG1_RL1/TSGR1_97/Docs/R1-1907399.zip

[23] Ericsson, R1-1705189, "Early data transmission for NB-IoT," 3GPP TSG RAN1 Meeting \#88bis, Spokane, USA, Apr 2017. [Online]. Available: https://www.3gpp.org/ftp/TSG_RAN/WG1_RL1/TSGR1_88b/Docs/R1-1705189.zip

[24] Ericsson, R1-1706161, "Early data transmission for MTC," 3GPP TSG RAN1 Meeting \#88bis, Spokane, USA, Apr 2017. [Online]. Available: https://www.3gpp.org/ftp/TSG_RAN/WG1_RL1/TSGR1_88b/Docs/R1-1706161.zip

[25] H. Malik, H. Pervaiz, M. Mahtab Alam, Y. Le Moullec, A. Kuusik and M. Ali Imran, "Radio Resource Management Scheme in NB-IoT Systems," IEEE Access, vol. 6, pp. 15051-15064, 2018, doi: 10.1109/ACCESS.2018.2812299.

[26] 3GPP TS 36.213, "Evolved Universal Terrestrial Radio Access (E-UTRA): Physical layer procedures (Rel-16)," version 16.3.0, pp. 548-551, Sep 2020. [Online]. Available: https://www.3gpp.org/ftp/Specs/archive/36_series/36.213/36213-g40.zip

[27] 3GPP TR 45.820, "Cellular system support for ultra-low complexity and low throughput Internet of Things (CIoT) Release 13," version 13.1.0, Nov 2015. [Online]. Available: https://www.3gpp.org/ftp/Specs/archive/45_series/45.820/45820-d10.zip

[28] ITU-R, Report M.2412-0, "Guidelines for evaluation of radio interface technologies for IMT-2020," Oct 2017. [Online]. Available: https://www.itu.int/dms_pub/itu-r/opb/rep/R-REP-M.2412-2017-PDF-E.pdf. 


\section{BIOGRAPHIES OF AUTHORS}

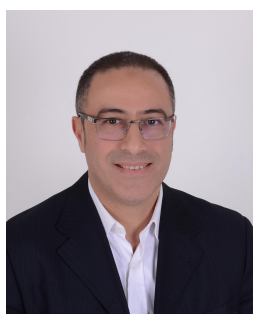

Adil Abou El Hassan received the B.Sc. degree in electrical engineering from Hassan II University (ENSET), Mohammedia, Morocco, in 1993 and the M.Sc. degree in electrical engineering from Mohammed V University (ENSET), Rabat, Morocco, in 2019. He is currently pursuing the Ph.D. degree at Mohammed First University (National School of Applied Sciences), Oujda, Morocco. His research interests include the Internet of Things (IoT), 5G wireless communication and networks, LPWAN technologies and resource management of wireless communications.

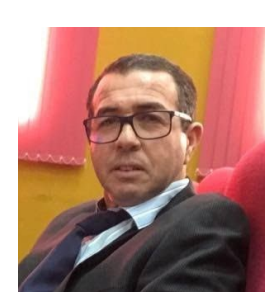

Abdelmalek El Mehdi received the Ph.D. degree in Electronics and telecommunications from the University Pierre \& Marie Curie of Paris on 2008. He is currently a Professor- researcher of electronics and automation at the national school of applied sciences (ENSAO), Mohammed First University in Morocco. His interests include modeling and control of advanced electrical power systems, design, application of power electronics and Telecommunications Engineering.

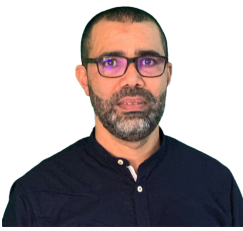

Mohammed Saber received his Ph.D. in Computer Science at Faculty of Sciences Oujda, in July 2012. He is currently an Associate professor in Computer Science (Network, Security and Systems) in National School of Applied Sciences (ENSAO) at Mohammed First University, Oujda, Morocco. His interests include Network Security (Intrusion Detection System, Evaluation of security components), Security in Mobile Ad Hoc Networks (MANET), Security of IoT and Robotics. 